\title{
Factores de riesgo de accidentes de tránsito en adolescentes
}

\author{
A Population-Based Study of Crashes Involving 16 and 17 Years Old Drivers: The Potencial Benefit of Graduated Driver Licensing \\ Restrictions. Cvijanovich N, Cook L, Mann N y col. Pediatrics 2001;107:632-637
}

\section{Objetivo}

Evaluar la efectividad potencial del programa de licencia de conducir con restricciones graduales (GDLR) revisando bases poblacionales de accidentes que involucraron conductores adolescentes (CA).

\section{Diseño}

Estudio sobre datos secundarios de Utah (EE.UU) provenientes de cuatro fuentes de registros: Accidentes de tránsito, egresos hospitalarios, egresos de Departamentos de Emergencia, Base de la División Licencias de Conducir.Período observado 1992-1996.

\section{Participantes}

Se consideraron dos subgrupos: adolescentes (16-17 años) y adultos (18-59 años) para la mayoría de las comparaciones realizadas.

\section{Medición de resultados principales}

El foco de interés primordial fue determinar la influencia potencial de "la intervención" (el programa GDLR) sobre los accidentes en el grupo adolescente. Se realizó un análisis descriptivo del accidente y los ocupantes y de la asociación entre población y severidad del accidente; fallas del conductor; uso del cinturón; horario; número de pasajeros. Se utilizó un modelo de análisis multivariable* para evaluar la asociación entre factores ambientales y accidentes comparando los dos subgrupos etarios.

\section{Resultados}

De la población entre 16-59 años con registro, el 5.8\% fueron CA y estuvieron involucrados en el $19 \%$ de los accidentes. Los accidentes con CA tuvieron 158 muertes. El 63\% eran acompañantes de CA;el $45 \%$ eran conductores.El $85 \%$ de todos los adolescentes muertos viajaban con CA. Los accidentes con CA representaron U\$S 11 millones en egresos hospitalarios relacionados sin considerar las salas de emergencia (cuya proyección agregaría 10 millones más), ni los cuidados domiciliarios, rehabilitación y otros. Comparando las características del accidente de CA vs adultos; el OR para accidentes nocturnos fue 1.31 [IC95\% 1.28-1.34]; un solo auto involucrado: OR 1.12 [IC95\% 1.09-1.16];víctimas fatales de todos los accidentes:OR 0.59 [0.48-0.73].Sin embargo la mortalidad con CA sigue siendo alta entre las 22 PM y 06 AM, pues el $19 \%$ de accidentes fatales que involucraron CA ocurrieron en este lapso. El riesgo de hospitalización o muerte del CA es mayor cuanto mayor es el número de pasajeros (OR 1.62 a 2.47 según sean 2 o 5 pasajeros). En cambio para los adultos los OR son de 1.25 a 1.83 en esa misma escala. El riesgo de recibir citaciones $\mathrm{p}$ 0 exceso de velocidad de CA vs adultos fue 1.43 [IC95\% 1.35-1.50] al igual que por manejo descuidado: 2.83 [IC95\% 2.44-3.27]. La presencia de pasajeros aumentó también este riesgo para CA casi un $100 \%$ en forma incremental con el número de acompañantes.

\section{Conclusión}

Los conductores adolescentes tienen mayor riesgo de protagonizar accidentes; alta fatalidad en horas nocturnas; cometen más errores; usan menos el cinturón;la presencia de pasajeros incrementa la severidad de los accidentes así como la mayor tendencia a ser citados por exceso de velocidad o manejo distraído. La presencia de un mayor de 21 años entre los acompañantes disminuye los riesgos. Todo esto provoca un significativo impacto económico.El presente estudio brinda soporte a la decisión de implementar un programa GDLR adaptado a las características de

Fuente de financiamiento:no referida.

\section{Comentario}

En otros estados y países 1-3 los programas GDLR han logrado disminuir accidentes entre 7-32\% 4-6. Estos programas consisten en liberar restricciones progresivamente a medida que los conductores adolescentes (CA) logran experiencia y madurez durante los 18 meses de duración desde la primera licencia transitoria otorgada7. Sus principales etapas consisten en ir acompañados por adultos con licencia, prohibir horarios nocturnos y evitar otros pasajeros. Este estudio describe la sobrerepresentación de los $\mathrm{CA}$ en los accidentes viales y su impacto económico. Si bien los CA protagonizan accidentes con menor severidad y fatalidad que los adultos, en Utah, restringir las horas de la noche tal vez evitaría $11 \%$ de accidentes y $19 \%$ de los fatales. La presencia de pasajeros con CA incrementa en conjunto casi $70 \%$ el riesgo de algún tipo de accidente8 lo mismo que su citación al tribunal de faltas en 2.5 veces. El $97.2 \%$ de los accidentes de CA ocurren solos o con acompañantes menores de 21 años. Si bien suelen viajar con menores de 21 años, esto no alcanza a descartar la influencia positiva de al menos un pasajero mayor 21 años sobre la conducta vial del CA y el uso de cinturón de seguridad de todos los ocupantes.

Algunas limitaciones del estudio son: el sesgo* en el reporte del uso del cinturón (auto reporte) y respecto a las citaciones, la animadversión natural de los policías para con los CA.Se desprende que las conductas de riesgo están fuertemente asociadas con la presencia de pares 9,10 y que las horas de la madrugada son las más peligrosas5,11. La mayor contribución a la muerte de adolescentes en accidentes viales se asocia a inexperiencia, impulsividad, inmadurez y distracción ${ }^{12,13}$. De alguna manera la implementación de GDLR ${ }^{14}$ tiende a corregir esta natural discapacidad transitoria inherente a esta etapa de la vida. Dada la abundante experiencia internacional nuestro país debiera revisar la edad y las restricciones en el otorgamiento de licencias de conducir. Médicos y padres debemos extremar el cuidado de riesgos controlables, porque aunque resulte extenuante, mantenerlos saludables siempre vale la pena: en este caso sobre el uso de cinturón de seguridad, horarios y condiciones prudentes para conducir.

\section{Dr. Virgilio Petrungaro [ Especialista en Clínica Médica.Director Médico.Instituto Cardiovascular de Buenos Aires. ]}

\footnotetext{
Referencias

1. Begg DJ, Langley JD, Reeder Al, The New Zealand graduated driver licensing system: teenagers' attitudes toward and experiencies with this car driver licensing system.Inj Prev. 1995;1:177-181 2. Boase P, Tasca L. Graduated Licensing System Evaluation.Interim Report.Ottawa, Ontario, Canada:Safety Policy Branch, Ministry of Transportation;1998.Report 410 SPB-98-101 3.Langley JD, Wagenaar AC, Begg DJ.An evaluation of the New Zealand graduated driver licensing system.Accid Anal Prev. 1996;28:139-146 4. Luciana Phebo, Ann M. Dellinger.Young driver involvement in fatal motor vehicle crashes and trends in risk behaviors, United States, 1988-95. Injury Prevention 1998;4:284-287. 5. Foss RD, Evenson KR. Effectiveness of graduated driver licensing in reducing motor vehicle crashes.Am J Prev Med. 1999;16(suppl):47-56

6. National Highway Traffic Safety Administration.Saving teenage lives: the case for graduated driver licensing. http://www.nhtsa.dot.gov/people/injury/newdriver/SaveTeens/toc.html 6. National Highway Traffic Safety Administration.Saving teenage lives: the case for graduated driver licensing.http://www.nhtsa.dot.gov/people/injury/newdriver/SaveTeens/toc.html
7. National Highway Traffic Safety Administration. Graduated Driver Licensing System for Young Novice Drivers. Washington, DC:US Department of Transportation, National Highway Traffic Safety Ad-

8. Chen L, Baker SP, Braver ER, et al. Carrying passengers as a risk factor for crashes fatal to 16 and 17 years old drivers. JAMA, March 22/29, 2000-Vol. 283, № 12:1578-1582. 9. Farrow JA. Young driver risk taking:a description of dangerous driving situations among 16-19 years old drivers. Int.j.Addict.1987;22:1255-1267.

10.Mc. Kenna FP, Waylen AE, Burkes ME.Male and Female Drivers, How different are they? Reading, England: University of Reading, AA Foundation for Road Safety Research; 1998.

11. Yang Mao, Jun Zhang, Glenn Robbins, et al.Factors affecting the severity of motor vehicle traffic crashes involving young drivers in Ontario. Injury Prevention 1997; 3:183-189.

12. National Highway Traffic Safety Administration. Fatality Analysis Reporting System, 1998. http:/www-fars.nhtsa.dot.gov/www/query.htlm. Accessed January 15, 2000.

13. Williams AF, Preusser DF, Ulmer RG, Weinstein HB. Characteristics of fatal crashes of 16 years old drivers: implications for licensure policies.J Public Health Policy. 1995;16:347-360.

14. Foss DF.Reducing fatal crash risk among teenaged drivers. Structuring an effective graduated licensing system. p1617-8.Editorials.
} 